\title{
Generalized Chern-Simons Gravity: Classical Formalism
}

\author{
Clisthenis $\mathbf{P}$ Constantinidis \\ Departamento de Física, Universidade Federal do Espírito Santo (UFES), Vitória, ES, Brazil \\ E-mail: cpconstantinidis@pq.cnpq.br
}

\section{Zui Oporto*†}

Departamento de Física, Universidade Federal do Espírito Santo (UFES), Vitória, ES, Brazil E-mail: azurnasirpalegmail.com

\section{Olivier Piguet}

Departamento de Física, Universidade Federal do Espírito Santo (UFES), Vitória, ES, Brazil

E-mail: opiguet @pq. cnpq.br

\begin{abstract}
We present the canonical analysis of a generalized formulation of $3 \mathrm{~d}$ gravity as a Chern-Simons gauge theory with a Barbero-Immirzi-like ambiguity. After a description of some general arguments and a brief introduction to the Chern-Simons formulation of $3 \mathrm{~d}$ gravity, we analyze a specific model with positive cosmological constant. Finally, it is discussed a gauge fixing strategy as a first step towards canonical quantization of the model.
\end{abstract}

4th International Conference on Fundamental Interactions -ICFI2010,

August 1-7, 2010

Viçosa, Brazil

\footnotetext{
* Speaker.

†Thanks to CLAF-CNPq for supporting this work
} 


\section{Introduction}

Since the discovery by Achucarro and Townsend [1] and the elaborated work of Witten [2], it is a sort of common sense to affirm that $3 \mathrm{~d}$ gravity and Chern-Simons (CS) gauge theory are equivalent, up to a total derivative (boundary terms), with Poincaré group being the underlying gauge group. However, the difference between the two theories is that in $3 \mathrm{~d}$ gravity the triad is restricted to being invertible, whereas no such restriction exists in CS theory. Thus, We can think about CS theory as an extension of $3 \mathrm{~d}$ gravity including singular metrics or, alternatively, think about $3 \mathrm{~d}$ gravity as a restricted version of CS theory. Questions regarding the role of non-invertible triad is far from being trivial [3].

A richer structure emerges by enlarging the local symmetry to the (anti) de Sitter group. In this case, besides the standard action for $3 \mathrm{~d}$ gravity, it is possible to construct an "exotic" action equivalent to the former at the level of classical field equations. This peculiarity of CS gravity was not unnoticed in original Witten's paper.

In a more recent context, there exists an analogy with a well established (although polemic) feature of Loop Quantum Gravity (LQG) knew as the Barbero-Immirzi parameter ambiguity [4, 5]. This analogy was studied in detail in [6], and represents the principal motivation of the present work.

The plan of the paper is as follows. In the next section, the basic tools to cast $3 \mathrm{~d}$ gravity with or without cosmological constant as a Chern-Simons gauge theory will be presented, and the appearance of the Barbero-Immirzi parameter will be explained. In Section 3 the main argument of the paper will be presented, in which we consider the positive cosmological constant model. The detailed canonical analysis of constrained theory will be developed. The last section is reserved to some comments about gauge fixing reduction and quantization of the model in the spirit of LQG, as well as the conclusions of the work.

\section{Gravity from Chern-Simons theory with Barbero-Immirzi-like ambiguity.}

To begin with, let $\mathscr{M}$ be an orientable three-dimensional manifold with a trivial tangent bundle. In addition, let $G$ be the gauge group and $\mathfrak{g}$ its Lie algebra equipped with a non-degenerate invariant quadratic form $\langle\cdot, \cdot\rangle$ (inner product on the Lie algebra).

The CS action is defined as

$$
S=-\frac{\kappa}{2} \int_{\mathscr{M}}\left\langle\mathrm{A}, \mathrm{dA}+\frac{2}{3} \mathrm{~A} \wedge \mathrm{A}\right\rangle
$$

where $\mathrm{A}=A_{\mu} \mathrm{d} x^{\mu}$ is a $\mathfrak{g}$-valued 1-form connection and $\kappa$ a dimensionless constant. ${ }^{1}$ The field equations read $F=0$, with $F \equiv d A+A \wedge A$ is, by definition, the field strength 2-form; this means that CS theory is topological with no truly propagating degrees of freedom. By construction, CS action is diffeomorphism invariant.

\footnotetext{
${ }^{1}$ In what follows Greek indices $\mu, v, \ldots$ run from 0 to 2 and Latin indices from the beginning of the alphabet $a, b, \ldots$ takes values 1,2 . Three dimensional Lorentz frame indices are denoted by Latin capital letters $I, J, \ldots$ running from 0 to 2. Our convention for the space-time metric is $\eta_{I J}=\operatorname{diag}(\sigma, 1,1)$. Where $\sigma= \pm 1$ allows us to switch between the Euclidian and Lorentzian cases, respectively.
} 
Next we assume $\mathscr{M}$ to be of topology $\Sigma \times \mathbb{R}$, where $\Sigma$ is a two-dimensional manifold representing physical space, and $\mathbb{R}$ the real line representing time. The Hamiltonian formalism can be achieved by splitting the connection into its temporal and spatial components: $A=A_{t} d x^{0}+A_{a} d x^{a}$. Replacing this into (2.1) the action can be written as

$$
S=-\frac{\kappa}{2} \int_{\mathbb{R}} \mathrm{d} x^{0} \int_{\Sigma}\langle\dot{\mathbf{A}}, \mathbf{A}\rangle+2\left\langle\mathrm{~A}_{t}, \mathbf{F}\right\rangle,
$$

where $\mathbf{A}=\mathrm{A}_{a} \mathrm{~d} x^{a}$ is the spatial connection and $\mathbf{F}=\mathrm{d} \mathbf{A}+\mathbf{A} \wedge \mathbf{A}$ the associated spatial field strength.

Three-dimensional gravity meets $C S$ theory when we choose as underlying gauge symmetry the Poincare group $I S O(1,2)$. In this case, the connection is written as $\mathrm{A}=e^{I} P_{I}+\omega^{I} J_{I}$, where $e$ is the triad and $\omega$ the spin connection, whereas $P_{I}$ and $J_{I}$ correspond to the generators of translations and rotations of $I S O(2,1)$, respectively. We can go further and include a positive (negative) cosmological constant $\Lambda$ by enlarging the gauge symmetry to the (anti) de Sitter $\operatorname{SO}(3,1)(\operatorname{SO}(2,2))$ group. In any case, the generators will satisfy the general Lie algebra given by $\left[J_{I}, J_{J}\right]=\varepsilon_{I J}{ }^{K} J_{K}$, $\left[J_{I}, P_{J}\right]=\varepsilon_{I J}{ }^{K} P_{K}$ and $\left[P_{I}, P_{J}\right]=\sigma \Lambda \varepsilon_{I J}{ }^{K} J_{K}$.

A special feature of the $S O(3,1)$ is the possibility to define two non-degenerate quadratic forms in the algebra. These are given by the Casimir invariants of the algebra

$$
\mathscr{C}=\eta^{I J} P_{I} J_{J} \quad \text { and } \quad \mathscr{C}^{\star}=\eta^{I J}\left(\frac{\sigma}{\Lambda} P_{I} P_{J}+J_{I} J_{J}\right) .
$$

The inner product defined by $\mathscr{C}$ is non degenerate for all $\Lambda$, whereas $\mathscr{C}^{\star}$ is non degenerate only for $\Lambda \neq 0$.

Starting with (2.1), we can write an action for each Casimir invariant form, these are,

$$
\begin{aligned}
S & =-\frac{\kappa}{2} \int_{\mathscr{M}}\left[\dot{\mathbf{e}} \omega+\dot{\omega} \mathbf{e}+2 e_{t}\left(\mathbf{R}+\Lambda \mathbf{e}^{2}\right)+2 \omega_{t} \mathbf{T}\right], \\
S^{\star} & =-\frac{\kappa}{2} \int_{\mathscr{M}}\left[\sigma \Lambda \dot{\mathbf{e}}+\dot{\omega} \omega+2 \sigma \Lambda e_{t} \mathbf{T}+2 \omega_{t}\left(\mathbf{R}+\Lambda \mathbf{e}^{2}\right)\right] .
\end{aligned}
$$

It can be recognized in $S$ the standard action for 3d gravity. On the other hand, $S^{\star}$ can be considered a kind of "exotic" $3 \mathrm{~d}$ gravity in the sense that, despite being inequivalent to the standard one, it shares the same field equations. It makes perfect sense to add the exotic action, with an arbitrary coefficient $\gamma$, to the standard action, so the general action would be, $S=S-\frac{1}{\gamma} S^{\star}$.

It is worth noticing that the equivalence can be established only at the level of field configurations, but this is not true at the level of the structure of the phase-space. In [6] this intriguing model was studied in detail within the context of LQG, where the appearing of $\gamma$ is compared with the arbitrariness of the Barbero-Immirzi parameter [5].

This completes our brief description of Chern-Simons formulation of 3d Gravity and the origin of the $\gamma$ parameter.

\section{Chern-Simons gravity with positive cosmological constant}

In what follows we will restrict the model to the $\Lambda>0$ sector. (The case of negative cosmological constant can be constructed analogously.) Also, we have kept open the possibility to switch 
between the Euclidian and Lorentzian theories by introducing the parameter $\sigma= \pm 1$, so the gauge group would be $S O(4)$ or $S O(3,1)$, respectively.

Let's start writing the spatial connection as $\mathbf{A}=\mathbf{A}_{1}^{i} K_{i}+\mathbf{A}_{2}^{i} L_{i}$, where $K_{i}$ are the generators of boosts and $L_{i}$ the generators of rotations of the $S O(3,1)(S O(4))$ group that satisfy the Lie algebra $\left[L_{i}, L_{j}\right]=\varepsilon_{i j}{ }^{k} L_{k},\left[L_{i}, K_{j}\right]=\varepsilon_{i j}{ }^{k} K_{k}$ and $\left[K_{i}, K_{j}\right]=\sigma \varepsilon_{i j}{ }^{k} L_{k}$. Here $i, j$, take values $1,2,3$ and are raised or lowered with the delta Kronecker $\delta_{i j}$.

The dictionary to restore the triad and spin connection is

$$
\begin{aligned}
K & =\left(J_{2},-J_{1}, P_{0} / \sqrt{\Lambda}\right), \quad L=\left(P_{2} / \sqrt{\Lambda},-P_{1} / \sqrt{\Lambda}, \sigma J_{0}\right) ; \\
\mathbf{A}_{1} & =\left(\omega^{2},-\omega^{1}, \sqrt{\Lambda} \mathbf{e}^{0}\right), \quad \mathbf{A}_{2}=\left(\sqrt{\Lambda} \mathbf{e}^{2},-\sqrt{\Lambda} \mathbf{e}^{1}, \sigma \omega^{0}\right) .
\end{aligned}
$$

As before, the non degenerate quadratic forms in the algebra are given by the two Casimirs of the group, then it can be shown that ${ }^{2}\langle\mathbf{A}, \mathbf{B}\rangle=\mathbf{A}_{1} \cdot \mathbf{B}_{2}+\mathbf{A}_{2} \cdot \mathbf{B}_{1}$ and $\langle\mathbf{A}, \mathbf{B}\rangle^{\star}=\sigma \mathbf{A}_{1} \cdot \mathbf{B}_{1}+\mathbf{A}_{2} \cdot \mathbf{B}_{2}$

With all these, the general action can be written as

$$
S=-\frac{\kappa}{2} \int\left[\dot{\mathbf{A}}_{1} \cdot\left(\mathbf{A}_{2}-\frac{\sigma}{\gamma} \mathbf{A}_{1}\right)+\dot{\mathbf{A}}_{2} \cdot\left(\mathbf{A}_{1}-\frac{1}{\gamma} \mathbf{A}_{2}\right)\right]-\mathscr{G}_{1}\left(\mathrm{~A}_{t 1}\right)-\mathscr{G}_{2}\left(\mathrm{~A}_{t 2}\right),
$$

where we are defining the smeared "constraints" (the reason of this denomination will be clear in a moment)

$$
\mathscr{G}_{1}(\varepsilon) \equiv \kappa \int \varepsilon \cdot\left[\mathbf{F}+\frac{\sigma}{2} \mathbf{A}_{1} \times \mathbf{A}_{1}-\frac{\sigma}{\gamma} \mathrm{DA}_{1}\right], \quad \mathscr{G}_{2}(\varepsilon) \equiv \kappa \int \varepsilon \cdot\left[\mathrm{D} \mathbf{A}_{1}-\frac{1}{\gamma}\left(\mathbf{F}+\frac{\sigma}{2} \mathbf{A}_{1} \times \mathbf{A}_{1}\right)\right],
$$

together with $\mathbf{F}=\mathrm{d} \mathbf{A}_{2}+\mathbf{A}_{2} \wedge \mathbf{A}_{2}$ and $\mathrm{D} \mathbf{A}_{1}=\mathrm{d} \mathbf{A}_{1}+\left[\mathbf{A}_{2}, \mathbf{A}_{1}\right]$.

Let's write gauge fields as two component vectors $A_{t}=\left(A_{t 1}, A_{t 2}\right)$ and $\mathbf{A}=\left(\mathbf{A}_{1}, \mathbf{A}_{2}\right)$. Now, if we introduce the matrix

$$
\Delta^{\alpha \beta}=\left(\begin{array}{cc}
-\sigma / \gamma & 1 \\
1 & -1 / \gamma
\end{array}\right),
$$

with $\alpha, \beta=1,2$, then (3.3) adopts the form of the standard CS action,

$$
S=-\frac{\kappa}{2} \int \Delta^{\alpha \beta}\left(\dot{\mathbf{A}}_{\alpha} \cdot \mathbf{A}_{\beta}+2 \mathrm{~A}_{t \alpha} \cdot \Omega_{\beta}\right),
$$

where $\Omega=\left(\mathrm{DA}_{1}, \mathbf{F}+\frac{\sigma}{2} \mathbf{A}_{1} \times \mathbf{A}_{1}\right)$ is the field strength associated with $\mathbf{A}$.

As before, it is convenient to define the smeared version of the constraints

$$
\mathscr{F}_{1}(\varepsilon) \equiv \kappa \int \varepsilon \cdot \mathrm{DA}_{1}, \quad \mathscr{F}_{2}(\varepsilon) \equiv \kappa \int \varepsilon \cdot\left(\mathbf{F}+\frac{\sigma}{2} \mathbf{A}_{1} \times \mathbf{A}_{1}\right) .
$$

The relation between the $\mathscr{G}$-base and the $\mathscr{F}$-base is simply $\mathscr{G}=\Delta \mathscr{F}$.

Applying the Dirac-Bergman's algorithm for constrained system [7], we obtain the symplectic structure of the phase-space described by the Dirac brackets

$$
\left\{\mathrm{A}_{a \alpha}^{i}(\mathbf{x}), \mathrm{A}_{b \beta}^{j}(\mathbf{y})\right\}_{D}=-\frac{1}{\kappa} \Delta_{\alpha \beta} \varepsilon_{a b} \delta^{i j} \delta^{3}(\mathbf{x}-\mathbf{y}) .
$$

\footnotetext{
${ }^{2}$ Since all group indices are contracted with the three dimensional metric $\delta_{i j}$, it is convenient adopting vector-like notation $\mathbf{A}^{i} \mathbf{B}_{i}=\mathbf{A} \cdot \mathbf{B},[\mathbf{A}, \mathbf{B}]=\mathbf{A} \times \mathbf{B}$, etc.
} 
From these relations we can see that half of the $A_{a \alpha}^{i}$ play the role of momenta, thus the original 24-dimensional phase-space would be reduced to the half.

The Hamiltonian would be

$$
H=\mathscr{F}\left(\mathrm{A}_{t}\right)+u_{\alpha} \chi^{\alpha}+v_{\alpha} \chi^{t \alpha}=\mathscr{G}_{1}\left(\mathrm{~A}_{t 1}\right)+\mathscr{G}_{2}\left(\mathrm{~A}_{t 2}\right)+u_{\alpha} \chi^{\alpha}+v_{\alpha} \chi^{t \alpha},
$$

where $\mathscr{F}\left(\mathrm{A}_{t}\right)=\Delta^{\alpha \beta} \mathscr{F}_{\alpha}\left(\mathrm{A}_{t \beta}\right)$ and $u_{\alpha}, v_{\alpha}$ stand for lagrange multipliers. From here we can see that the Hamiltonian is fully constrained. The algebra of the constraints closes under Dirac brackets as can be see from $\left\{\mathscr{F}(\varepsilon), \mathscr{F}\left(\varepsilon^{\prime}\right)\right\}_{D}=\mathscr{F}\left(\varepsilon \times \varepsilon^{\prime}\right)$. This algebra adopts a more suggestive form in the $\mathscr{G}$-basis,

$$
\left\{\mathscr{G}_{1}(\varepsilon), \mathscr{G}_{1}\left(\varepsilon^{\prime}\right)\right\}_{D}=\sigma \mathscr{G}_{2}\left(\varepsilon \times \varepsilon^{\prime}\right), \quad\left\{\mathscr{G}_{1}(\varepsilon), \mathscr{G}_{2}\left(\varepsilon^{\prime}\right)\right\}_{D}=\mathscr{G}_{1}\left(\varepsilon \times \varepsilon^{\prime}\right), \quad\left\{\mathscr{G}_{2}(\varepsilon), \mathscr{G}_{2}\left(\varepsilon^{\prime}\right)\right\}_{D}=\mathscr{G}_{2}\left(\varepsilon \times \varepsilon^{\prime}\right) .
$$

We can recognize here the structure of $\mathfrak{s o}(3,1)(\mathfrak{s o}(4))$ Lie algebra, in total agreement with the fact that in Dirac-Bergman formalism for constrained systems, first class constraints generate local gauge transformations.

The infinitesimal gauge transformations generated by the constraints are

$$
\begin{aligned}
\left\{\mathscr{G}_{1}(\varepsilon), \mathbf{A}_{1}\right\}_{D} & =\mathrm{D} \boldsymbol{\varepsilon}, \quad\left\{\mathscr{G}_{1}(\varepsilon), \mathbf{A}_{2}\right\}_{D}=\sigma \varepsilon \times \mathbf{A}_{1} ; \\
\left\{\mathscr{G}_{2}\left(\varepsilon^{\prime}\right), \mathbf{A}_{1}\right\}_{D} & =\varepsilon^{\prime} \times \mathbf{A}_{1}, \quad\left\{\mathscr{G}_{2}\left(\varepsilon^{\prime}\right), \mathbf{A}_{2}\right\}_{D}=\mathrm{D} \varepsilon^{\prime} .
\end{aligned}
$$

These relations look like the infinitesimal gauge transformations of a BF-theory with cosmological constant, where $\mathbf{A}_{1}$ plays the role of the $B$-field. This makes sense because of the fact that $3 \mathrm{~d}$ gravity can be formulated also as a topological BF-theory.

The gauge transformations described above are related, on-shell, with local diffeomorphism. This can be shown if we apply the Lie derivative to the gauge fields

$$
£_{\xi}\left(\mathbf{A}_{1}, \mathbf{A}_{2}\right)=\left(\mathrm{D}\left(\iota_{\xi} \mathbf{A}_{1}\right)+\left(\iota_{\xi} \mathbf{A}_{2}\right) \times \mathbf{A}_{1}, \mathrm{D}\left(\iota_{\xi} \mathbf{A}_{2}\right)+\sigma\left(\iota_{\xi} \mathbf{A}_{1}\right) \times \mathbf{A}_{1}\right) \quad+\text { field equations, }
$$

with $£=\mathrm{d} l_{\xi}+l_{\xi} \mathrm{d}$ the Lie derivative. By comparison with (3.11) and (3.12) we can identify infinitesimal gauge transformations with parameters $\left(\varepsilon, \varepsilon^{\prime}\right)=\iota_{\xi}\left(\mathbf{A}_{1}, \mathbf{A}_{2}\right)$.

We can ask ourselves for the constraint algebra written in the more familiar $\mathscr{F}$-basis. This can be achieved by inverting $\mathscr{G}=\Delta \mathscr{F}$ from which we get $\mathscr{F}=\Delta^{-1} \mathscr{G}$, but it is useful to write instead the normalized basis $\tilde{\mathscr{F}}=-(\operatorname{det} \Delta) \Delta^{-1} \mathscr{G}$. With this, a direct calculation shows that

$$
\begin{aligned}
& \left\{\tilde{\mathscr{F}}_{1}(\varepsilon), \tilde{\mathscr{F}}_{1}\left(\varepsilon^{\prime}\right)\right\}_{D}=\tilde{\mathscr{F}}_{1}\left(\varepsilon \times \varepsilon^{\prime}\right)+\frac{1}{\gamma} \tilde{\mathscr{F}}_{2}\left(\varepsilon \times \varepsilon^{\prime}\right) \\
& \left\{\tilde{\mathscr{F}}_{1}(\varepsilon), \tilde{\mathscr{F}}_{2}\left(\varepsilon^{\prime}\right)\right\}_{D}=\tilde{\mathscr{F}}_{2}\left(\varepsilon \times \varepsilon^{\prime}\right)+\frac{\sigma}{\gamma} \tilde{\mathscr{F}}_{1}\left(\varepsilon \times \varepsilon^{\prime}\right) \\
& \left\{\tilde{\mathscr{F}}_{2}(\varepsilon), \tilde{\mathscr{F}}_{2}\left(\varepsilon^{\prime}\right)\right\}_{D}=\sigma\left(\tilde{\mathscr{F}}_{1}\left(\varepsilon \times \varepsilon^{\prime}\right)+\frac{1}{\gamma} \tilde{\mathscr{F}}_{2}\left(\varepsilon \times \varepsilon^{\prime}\right)\right) .
\end{aligned}
$$

This time, it results less evident that the underlying symmetry corresponds to the $S O(3,1)$ group but, serves us to compare this algebra with the one reported in [6]. It is important to note that in our model we are mixing the components e and $\omega$ according to relations (3.1) and (3.2). 


\section{Concluding remarks.}

The origin of the Babarbero-Immirzi ambiguity $\gamma$ in Chern-Simons formulation of 3d Gravity comes from the fact that it is possible to define two inequivalent inner products in the algebra of the $S O(3,1)$ group. Let's notice, however, that in $4 \mathrm{~d}$ gravity the gauge group corresponds to local Lorentz rotations, whereas in $3 \mathrm{~d}$ gravity corresponds to local Lorentz rotations and local translation. Therefore, it is expected a qualitative difference of the meaning of $\gamma$ in the $3 \mathrm{~d}$ and $4 \mathrm{~d}$ gravity theories.

We elaborated a detailed analysis of the $\Lambda>0$ sector of the theory. The case $\Lambda<0$ is not quite different, but technical difficulties to quantize the model are expected because of the noncompactness of the $S O(2,2)$ group.

After applying the Dirac-Bergman formalism for constrained systems, we ended with a first class Hamiltonian (3.9) together with the symplectic structure (3.8). It was shown that first class constraints generate local gauge transformations which at the same time are equivalent, on-shell, to local diffeomorphisms.

The analogy between $3 \mathrm{~d}$ CS gravity with $\Lambda>0$ and $4 \mathrm{~d}$ gravity suggest us to apply the methods of LQG to quantize the theory. In spite of CS theory is considered a well-known toy model in gauge field theory, the extension of the LQG machinery to quantize this theory was not fully exploited. An interesting example of LQG-like quantization of CS theory was developed in [8] for a compact gauge group. There is not a generally accepted method for non-compact groups. In $4 \mathrm{~d}$ gravity the quantization is accomplished by partial gauge fixing of the non-compact group[4] such that the residual symmetry turns to be the $S O(3)$ group. It is interesting asking about a gauge-fixing strategy to seek out for the quantization of the generalized CS gravity considered in the present work [9].

\section{References}

[1] A. Achucarro and P. K. Townsend, A Chern-Simons Action for Three-Dimensional anti-De Sitter Supergravity Theories, Phys. Lett. B 180, 89 (1986).

[2] E. Witten, (2+1)-Dimensional Gravity as an Exactly Soluble System, Nucl. Phys. B 311, 46 (1988).

[3] H. J. Matschull, On the relation between $2+1$ Einstein gravity and Chern-Simons theory, Class. Quant. Grav. 16, 2599 (1999) [arXiv:gr-qc/9903040].

[4] A. Ashtekar and J. Lewandowski, Background independent quantum gravity: A status report, Class. Quant. Grav. 21, R53 (2004) [arXiv:gr-qc/0404018].

[5] C. Rovelli, Loop quantum gravity: the first twenty five years, arXiv:1012.4707 [gr-qc].

[6] V. Bonzom and E. R. Livine, A Immirzi-like parameter for 3d quantum gravity, Class. Quant. Grav. 25, 195024 (2008) [arXiv:0801.4241 [gr-qc]].

[7] M. Henneaux and C. Teitelboim, Quantization of gauge systems, Princeton, USA: Univ. Pr. (1992) $520 \mathrm{p}$

[8] C. P. Constantinidis, G. Luchini and O. Piguet, The Hilbert space of Chern-Simons theory on the cylinder. A Loop Quantum Gravity approach, Class. Quant. Grav. 27, 065009 (2010) [arXiv:0907.3240 [gr-qc]].

[9] C. P. Constantinidis, Z. Oporto and O. Piguet, (work in progress). 